\title{
Finger puzzles
}

\author{
R. W. Schmitt $\dagger$
}

Department of Physical Oceanography, Woods Hole Oceanographic Institution, Woods Hole, MA 02543, USA

Salt fingers are a form of double-diffusive convection that can occur in a wide variety of fluid systems, ranging from stellar interiors and oceans to magma chambers. Their amplitude has long been difficult to quantify, and a variety of mechanisms have been proposed. Radko \& Smith (J. Fluid Mech., this issue, vol. 692, 2012, pp. 5-27) have developed a new theory that balances the basic growth rate with that of secondary instabilities that act on the finite amplitude fingers. Their approach promises a way forward for computationally challenging systems with vastly different scales of decay for momentum, heat and dissolved substances.

Key words: double diffusive convection, instability, ocean processes

\section{Introduction}

Double-diffusive instabilities depend on a difference between the molecular diffusivities of two constituents and a gravitationally unstable distribution of one of them. The canonical example is 'salt fingers', wherein a top-heavy distribution of salt concentration is stabilized by the temperature profile. Heat is conducted about 100 times faster in water than salt diffuses, so small warm salty perturbations can lose more heat than salt, thus becoming denser and sinking, while cold fresh anomalies gain more heat than salt, becoming more buoyant and rising. The resulting flow consists of upgoing and downgoing columns of fluid in a self-assembled counterflow heat exchanger (as shown in the figure by the title from Krishnamurti 2009). The theory behind the basic salt-finger instability was developed over 50 years ago by Stern (1960). However, progress in understanding salt-finger equilibration has been slow. The limits on finger amplitude are controlled by nonlinear instabilities and their dynamics have remained elusive, in part because of the large range of scales involved in the fully developed salt-finger convection. Salt fingers have remained a challenging puzzle, ever since W. S. Jevons and Lord Rayleigh performed saltfinger experiments in 1857 and 1880 but failed to understand them (Schmitt 1995). New advances in analytical and numerical modelling reported by Radko \& Smith (2012, this issue) are finally leading to progress in quantifying the fluxes due to fingers. Since recent oceanographic tracer data has confirmed that salt fingers are very significant vertical mixing agents in the tropical oceans (Schmitt et al. 2005), there is an immediate need to advance the understanding of their dynamics. In addition, double-diffusive convection is now known to be important in a variety of other

$†$ Email address for correspondence: rschmitt@whoi.edu 


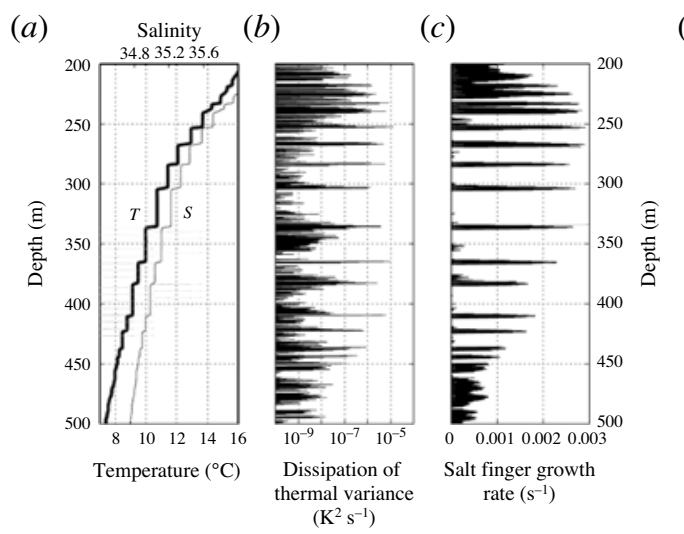

(d)

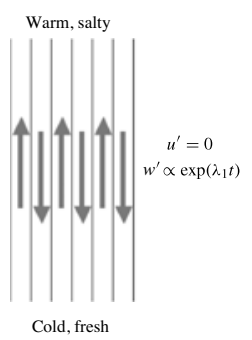

(e)

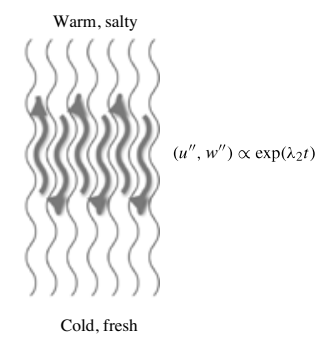

FIGURE 1. (a) Vertical profiles of temperature and salinity in the depth range of 200-500 m in the main thermocline of the tropical Atlantic ocean east of Barbados. $(b)$ Vertical profile of the dissipation rate of thermal variance as determined from microscale temperature gradients. (c) The theoretical growth rate of salt fingers calculated from the local vertical gradients of temperature and salinity and the seawater viscosity, thermal conductivity and salt diffusivity (from Schmitt et al. 2005, used with permission). (d,e) Schematic diagram illustrating development of primary salt-finger instabilities in a form of vertical elevator modes $(d)$ and their ultimate equilibration by secondary instabilities $(e)$ acting to distort fingers, thereby reducing the vertical transport of heat and salt. Radko \& Smith (2012) hypothesize that the equilibration occurs when the growth rates of primary (denoted by single primes) and secondary instabilities (double primes) are comparable.

systems from stellar interiors to magma chambers (Schmitt 1983), cases with vastly different Prandtl numbers and diffusivity ratios. Radko \& Smith (2012) have made a start on a consistent theory across such variations in fluid properties.

\section{Overview}

While the conditions for the initial growth of salt fingers are well understood, determining the processes that limit their magnitude has long proved difficult. Stern (1969) suggested that groups of fingers can feed internal wave instabilities when they reach sufficient amplitude. The motivation for this theory came from laboratory experiments that showed internal waves amplifying in a field of fingers in a uniform gradient situation. The waves presaged the onset of larger scale convective overturning that led to the formation of alternating mixed layers and gradient regions in what came to be called a thermohaline staircase. Both fingers and a mixed layer can be seen in laboratory experiments of Krishnamurti (2009) (as shown in the picture displayed near the title of this article) and an oceanic salt-finger staircase can be seen in figure 1( $a$ ).

Stern derived a criterion involving the ratio of the vertical buoyancy flux, the viscosity and the vertical buoyancy gradient: the so-called Stern number $A$ :

$$
A=\frac{\alpha F_{T}-\beta F_{S}}{\nu\left(\alpha \bar{T}_{z}-\beta \bar{S}_{z}\right)},
$$

where $F_{T}$ and $F_{S}$ are the temperature and salinity fluxes, $\bar{T}_{z}$ and $\bar{S}_{z}$ are the mean vertical temperature and salinity gradients, $\alpha$ and $\beta$ are the thermal expansion and 
haline contraction coefficients and $v$ is the kinematic viscosity. Also, Kunze (1987) considered the shear between adjacent upgoing and downgoing fingers and proposed a Richardson-type criterion for their equilibration, equivalent to Stern's constraint.

While parameter $A$ was an order-one number in the heat-salt case, a persistent stream of indirect evidence caused some doubt in the relevance of the Stern-Kunze arguments. For instance, laboratory experiments in which salt and sugar replace heat and salt as diffusive substances consistently yielded very low values of $A$. Another concern was expressed by Holyer (1984) who explored even finer-scale instabilities, developing on the strong horizontal gradients between fingers. Her theoretical analysis, supported by simulations in Shen (1995) and Radko \& Stern (1999), revealed that these secondary instabilities are similar to lateral intrusions and can potentially equilibrate the growth of primary salt fingers. Holyer's small-scale modes grow faster than the collective instabilities, seem to describe the turbulent appearance of fully developed fingers much better (again see the picture near the title) and, most importantly, are not controlled by $A$. Salt-finger equilibration in general and the relevance of the Stern number constraint in particular have remained among the most intriguing and controversial subjects in double-diffusive convection theory. However, the work reported by Radko \& Smith (2012) appears to resolve the long-standing controversy by sorting out the basic physics behind salt-finger amplitude equilibration and guiding us to a new understanding of these issues.

Radko \& Smith explore how the secondary instabilities can limit the growth of fingers. They argue that the limiting amplitude will be reached when the background finger growth rate is matched by the amplitude-dependent growth rate of the secondary instabilities, as illustrated in figures $1(d)$ and $1(e)$. They are guided in this development by careful numerical simulations, which are now made possible by computational advances. The balance between primary and secondary instabilities is simply presented in the relation:

$$
\lambda_{2}=C \lambda_{1},
$$

where $\lambda_{1}$ is the linear growth rate of the fingers as a function of the vertical property gradients and the molecular parameters, $\lambda_{2}$ is the growth rate of the secondary instabilities and $C$ is a dimensionless order one constant. The growth of the secondary instabilities is dependent on the amplitude of the fingers and the constant $C$ describes the relative efficiency of the two competing instabilities. Thus, the larger the growth rate of primary instabilities, the larger is the salt-finger amplitude.

There may already be some observational support for such a theory. That is, in addition to the thermohaline staircase, figures $1(a)-(c)$ show that the dissipation rate of thermal variance (a measure of the flux) peaks at every salt-finger interface in the oceanic staircases, as does the theoretical finger growth rate. The logarithm of the dissipation rate shows a strong correlation with the growth rate of the fingers, as determined from the local heat and salt stratification. These oceanographic data support a role for the growth rate in establishing the finger amplitude. Importantly, the model of Radko \& Smith (2012) suggests that mixing coefficients will decrease with increasing density ratio as observed in experiments and unlike the Stern and Kunze models.

Radko and Smith perform both two-dimensional and three-dimensional numerical simulations to evaluate $C$. It would not be surprising for $C$ to be a function of density ratio, but it appears to be well approximated by a constant value over a wide range of density ratios, Prandtl numbers and diffusivity ratios. The only exception is the parameter regime of the laboratory sugar-salt experiments, for which the variability 
in $C$ is attributed to an order-one diffusivity ratio. The overall success of the growth rate balance theory is an indication that a way forward has been defined for estimating finger amplitudes in the computationally challenging astrophysical case, characterized by huge differences in dissipation scales for momentum, heat and 'salt'.

\section{Future}

While the new theory of Radko \& Smith (2012) promises to resolve the finger amplitude challenge, there remains a host of unresolved problems in double diffusion. Mechanisms controlling the formation of thermohaline staircases (e.g. figure 1a) based on variations in the heat/salt flux ratio have been proposed by $\operatorname{Radko}(2003,2005)$, but remain unverified by laboratory experiments or field observations. The interaction of salt fingers with the larger scales of motion in other systems (stars, liquid metal and magmas) should be quite different, given the flux ratio dependencies predicted by linear theory. There remains the intriguing possibility that speckling in metal alloys and columnar jointing (basalt columns) are a signature of salt-finger convection that has been frozen into the solidified material. Recently, Hage \& Tilgner (2010) have reported the surprising discovery of fingers in haline convection, with the application of just a very small stable temperature gradient. This is a parameter regime where fingers were not expected, and large-scale convection was presumed to dominate. The field of double diffusion is rich with unresolved problems in the oceanographic heat-salt case and in the wide variety of other fluid systems susceptible to fingering.

\section{References}

Hage, E. \& Tilgner, A. 2010 High Rayleigh number convection with double diffusive fingers. Phys. Fluids 22, 076603.

Holyer, J. Y. 1984 The stability of long, steady, two-dimensional salt fingers. J. Fluid Mech. 147, $169-185$.

KRISHNAMURTI, R. 2009 Heat, salt and momentum transport in a laboratory thermohaline staircase. J. Fluid Mech. 638, 491-506.

KUnZE, E. 1987 Limits on growing, finite-length salt fingers: a Richardson number constraint. J. Mar. Res. 45, 533-556.

RADKo, T. 2003 A mechanism for layer formation in a double-diffusive fluid. J. Fluid Mech. 497, $365-380$.

RADKo, T. 2005 What determines the thickness of layers in a thermohaline staircase? J. Fluid Mech. 523, 79-98.

RADKo, T. \& SMith, D. 2012 Equilibrium transport in double-diffusive convection. J. Fluid Mech. 692, 5-27.

RAdko, T. \& Stern, M. E. 1999 Salt fingers in three dimensions. J. Mar. Res. 57, 471-502.

SCHMitT, R. W. 1983 The characteristics of salt fingers in a variety of fluid systems, including stellar interiors, liquid metals, oceans, and magmas. Phys. Fluids 26, 2373-2377.

Schmitt, R. W. 1995 The salt finger experiments of Jevons (1857) and Rayleigh (1880). J. Phys. Oceanogr. 25, 8-17.

Schmitt, R. W., Ledwell, J. R., Montgomery, E. T., Polzin, K. L. \& Toole, J. M. 2005 Enhanced diapycnal mixing by salt fingers in the thermocline of the tropical Atlantic. Science 308, 685-688.

SHEN, C. Y. 1995 Equilibrium salt-fingering convection. Phys. Fluids 7, 706-717.

StERn, M. E. 1960 The 'salt fountain' and thermohaline convection. Tellus 12, 172-175.

STERN, M. E. 1969 Collective instability of salt fingers. J. Fluid Mech. 35, 209-218. 